shows ischaemic changes, myocardial enzymes normal, coronary angiography showed at least a coronary artery stenosis degree $>70 \%$. The normal control group, there are 22 cases, 12 males and 10 females, mean age $(60.8 \pm 9.4)$ years of age. Selection criteria: chest pain, ECG and myocardial enzymes normal, coronary angiography showed coronary artery no stenosis. AMI and UAP patients' blood were taken $5 \mathrm{ml}$ immediately after admission, the normal control group blood were taken $5 \mathrm{ml}$ in the next morning. The blood serum was obtained after centrifugated on the same day and placed in $-20^{\circ} \mathrm{C}$ in refrigerator to preserve. Plasma concentrations of TNF- $\alpha$, IFN- $\gamma$, oxLDL, MMP-9 were measured in a day. Using enzyme-linked immunosorbent test (ELISA) test, kit were bought from Adlitteram Dignostic Laboratories, Inc in USA. The operating instructions strictly according to the kit. To read each hole OD value through the length $450 \mathrm{~nm}$ microplate. The sample values were taken into four parameters $\mathrm{OD}$ fitting formulae, calculated samples density. Use SPSS14.0 statistical software applications to analyse, measurement data using $\bar{x} \pm s$, measurement data use t-test, count data using $\chi^{2}$ test, $p<0.05$ for significant difference. Many factors applicate Logistic model do correlation analysis.

Results The plasma level of TNF- $\alpha$ in AMI group and UAP group, no statistical significance $(p>0.05)$, AMI group compared with the control group the serum levels of TNF- $\alpha$ increased significantly $(p<0.05)$, UAP group compared with the control group the serum levels of TNF- $\alpha$ increased significantly $(p<0.05)$. The plasma level IFN- $\gamma$ AMI group compared with UAP group, no statistical significance ( $p>0.05)$, AMI group compared with the control group the serum levels of IFN- $\gamma$ increased significantly $(p<0.05)$, UAP group compared with the control group the serum levels of IFN- $\gamma$ increased significantly $(p<0.05)$. MMP-9 plasma levels AMI group compared with UAP group the serum levels of MMP-9 increased significantly ( $<<0.05)$, AMI group compared with the control group the serum levels of MMP-9 increased significantly $(p<0.05)$, UAP group compared with the control group the serum levels of MMP-9 increased significantly $(p<0.05)$. The plasma levels of oxLDL AMI group compared with UAP group, no statistical significance $(p>0.05)$. AMI group compared with the control group the serum levels of oxLDL increased significantly $(p<0.05)$. UAP group compared with the control group the serum levels of oxLDL increased significantly $(\mathrm{p}<0.05)$.

Conclusion The plasma levels of TNF- $\alpha$, IFN- $\gamma$, MMP-9 and oxLDL in patients with acute coronary syndrome are significant positive correlation. The plasma levels of TNF- $\alpha$, IFN- $\gamma$, MMP-9 and oxLDL can be used to determine the nature of coronary atherosclerotic plaques. The plasma levels of MMP-9 increased hints plaques instability, Also can be used as an important biochemical monitoring index about the risk stratification of coronary artery disease.
Method From July 2008 to March 2010, intra-renal arterial digital subtraction angiography was performed in 302 patients who underwent coronary angiography. The incidence of $\geq 50 \%$ unilateral or bilateral renal artery stenosis were investigated in the population of the patients without and with CHD treating with various therapeutic regimens as following. By evaluating the coronary angiogram, the patients were not diagnosed to have $\mathrm{CHD}$ with less than $50 \%$ diameter stenosis of coronary artery; CHD was defined as narrowing of the appropriate lumen of $\geq 50 \%$; the procedure of $\mathrm{PCI}$ were performed in the patients with more than or equal to $70 \%$ stenosis; the CABG had been proposed in patients with left main coronary artery lesions, left main equivalent, diffuse triple coronary artery lesions, two-vessel disease with significant proximal left anterior descending $\mathrm{CAD}$. the intra-renal arterial digital subtraction angiography was performed, the renal artery stenosis was evaluated by three interventional cardiologists. RAS was defined as narrowing of the appropriate lumen of $\geq 50 \%$.

Results In 302 patients, 47 patients (15.56\%) had not been diagnosed to have CHD; 255 (84.44\%) to have CHD, of these patients with CHD, 80 patients $(26.49 \%)$ were not indicated for PCI, 24 $(7.95 \%)$ had been performed the procedure of stent implantation, $151(50 \%)$ proposed to have CABG. The incidence rates of renal artery stenosis were $6.38 \%(3 / 47)$ in patients without $\mathrm{CHD}$ and $40 \%$ $(102 / 302)$ in patients with CHD $(p=0.00087)$. In the four various therapeutic regimen groups, the incidence rates of RAS were $6.38 \%$ $(3 / 47)$ in the patients without CHD, 35\% (28/80) in the patients who were not indicated for PCI, $12.5 \%(3 / 24)$ in the patients performed stent implantation, and $47.02 \%(71 / 151)$ in the patients proposed to CABG. the incidence of RAS of the patients performed stent implantation was significantly lower than the patients who were not indicated for PCI $(p=0.035)$ and the patients proposed to CABG $(p=0.001)$. The age of was significantly older in patients with RAS than without RAS $(\mathrm{p}<0.0005)$. The morbidity rate of Diabetes (37.14\% and $25.38 \%, p=0.033)$, Cerebral infarction $(24.76 \%$ and 9.65\%, $\mathrm{p}<0.0005)$ and $\mathrm{CHD}(97.14 \%$ and $77.67 \%, \mathrm{p}<0.0005)$ were significantly higher in patients with RAS than without RAS. There is trend that more patients with Grade 3 hypertension in RAS group than normal renal artery group ( $65.71 \%$ vs $55.84 \%, \mathrm{p}=0.096)$. There were more patients with very high risk factors for cardiovascular diseases in RAS group than normal renal artery group (90.48\% vs $78.68 \%, p=0.01)$.

Conclusion The incidence of RAS was $6.38 \% \%$ in patients without CHD and $40 \%$ in patients with CHD. The incidence of RAS of the patients who were not indicated for PCI or proposed to CABG was higher than the patients performed stent implantation and patients without CHD. the age, Diabetes, Cerebral infarction, CHD, Grade 3 hypertension, and very high risk factors for cardiovascular diseases are the risk factors for RAS.

\section{Clinical and Research Medicine: Interventional Cardiology e0465 THE INCIDENCE OF RENAL ARTERY STENOSIS IN THE PATIENTS WITH CHD TREATING WITH VARIOUS THERAPEUTIC REGIMENS}

doi:10.1136/hrt.2010.208967.465

Feng Liang, Dayi Hu, Mingying Wu, Tianchang Li, Chuzhong Tang, Jiyun Wang, Changlin Lu. Department of Cardiology, Daxing Hospital, Capital University of Medical Science, Cardiac Center Beijing People's Hospital, Beijing, China

Introduction Renal artery stenosis (RAS) is one aspect of a multiterritory atherosclerotic disease. This study was to evaluate the incidence of RAS in the patients with CHD treating with various therapeutic regimen.

\section{C0466 VARIOUS THERAPEUTIC PROPORTION OF THE PATIENTS WITH TYPE 2 DIABETES MELLITUS UNDERGONE CORONARY ANGIOGRAPHY IN THE ERA OF DEVELOPMENT IN MSCT}

doi:10.1136/hrt.2010.208967.466

Feng Liang, Dayi Hu, Mingying Wu, Tianchang Li, Chuzhong Tang, Jiyun Wang, Changlin Lu. Department Of Cardiology, Daxing Hospital, Capital University Of Medical Science, Beijing, China

Introduction Diabetic patients are prone to a diffuse and rapidly progressive form of atherosclerosis, which increases their likelihood of requiring evascularization. This study was to examine the various therapeutic proportion of the population of the patients with type 2 diabetes mellitus undergone coronary angiography in the era of development in MSCT in the real clinical practice. 